\title{
Estimates of Scaling Violations for Pure SU(2) LGT
}

\author{
Bernd Berg ${ }^{1}$ and David Clarke ${ }^{1, \star}$ \\ ${ }^{1}$ Florida State University Department of Physics, Tallahassee, FL 32306
}

\begin{abstract}
We investigate the approach of pure SU(2) lattice gauge theory with the Wilson action to its continuum limit using the deconfining transition, Lüscher's gradient flow [1], and the cooling flow $[2,3]$ to set the scale. Of those, the cooling flow turns out to be computationally most efficient. We explore systematic errors due to use of three different energy observables and two distinct reference values for the flow time, the latter obtained by matching initial scaling behavior of some energy observables to that of the deconfining transition. Another important source of systematic errors are distinct fitting forms for the approach to the continuum limit. Besides relying in the conventional way on ratios of masses, we elaborate on a form introduced by Allton [4], which incorporates asymptotic scaling behavior. Ultimately we find that, though still small, our systematic errors are considerably larger than our statistical errors.
\end{abstract}

\section{Introduction}

Tests of the Standard Model, and New Physics searches require precise predictions of physical observables and accurate estimates of systematic uncertainty. Since scale setting is a source of uncertainty, it is useful to investigate scales that can achieve small statistical error bars, and to estimate the systematic error picked up from the choice of scale and the choice of fitting form for continuum limit extrapolations. Pure SU(2) gauge theory is a good testing ground for new scales because one can generate large statistics with relatively modest CPU resources.

Here we summarize the results [5] of a study of pure SU(2) LGT with the Wilson action

$$
S=\beta \sum_{\square}\left(1-\frac{1}{2} \operatorname{Tr} U_{\square}\right), \quad \beta=\frac{4}{g^{2}},
$$

where $g$ is the bare coupling constant and the summation runs over all plaquettes. We parameterize lattice expectation values of plaquette matrices by

$$
\left\langle U_{\square}\right\rangle_{L}=a_{0} \mathbf{1}+i \sum_{i=1}^{3} a_{i} \sigma_{i} .
$$

With this parameterization we use three definitions of the energy density:

$$
E_{0} \equiv 2\left(1-a_{0}\right), \quad E_{1} \equiv \sum_{i=1}^{3} a_{i}^{2}, \quad \text { and } \quad E_{4} \equiv \frac{1}{16} \sum_{i=1}^{3}\left(a_{i}^{u l}+a_{i}^{u r}+a_{i}^{d l}+a_{i}^{d r}\right)^{2},
$$

${ }^{\star}$ Speaker, e-mail: dclarke@hep.fsu.edu 
where $E_{4}$ is Lüscher's energy density, which averages over the four plaquettes attached to each site $x$ in a fixed $\mu v, \mu \neq v$ plane; the superscripts of $a_{i}$ stand for up $(u)$, left $(l)$, right $(r)$, and down $(d)$ with respect to $x$ (drawn in figure 1 of [1]). All definitions become $\sim F_{\alpha \beta} F_{\alpha \beta}$ in the continuum limit.

Configurations are obtained by Markov chain Monte Carlo (MCMC) simulations with Monte Carlo plus Overrelaxation (MCOR) updating. One MCOR sweep updates each link once in a systematic order with the Fabricius-Haan-Kennedy-Pendleton $[6,7]$ heatbath algorithm and, in the same systematic order, twice by overrelaxation [8]. Using checkerboard coding [9] and MPI Fortran, parallel updating of sublattices is implemented.

\section{Reference scales}

\subsection{Deconfinement scale}

The first scale investigated is the deconfinement scale. It is used to guide our choice of target values for the other scales. To calculate this scale we perform simulations on $N_{s}^{3} N_{\tau}$ lattices and estimate pseudocritical coupling constants $\beta_{c}\left(N_{s}, N_{\tau}\right)$ by reweighting the Polyakov loop susceptibility

$$
\chi(\beta)=\frac{1}{N_{s}^{3}}\left(\left\langle P^{2}\right\rangle-\langle|P|\rangle^{2}\right), \quad P=\sum_{\vec{x}} \prod_{x_{4}} U\left(\vec{x}, x_{4}\right)
$$

to nearby $\beta$ values and finding the maximum. Critical coupling constants $\beta_{c}\left(N_{\tau}\right)=\beta_{c}\left(\infty, N_{\tau}\right)$ are then extrapolated using three-parameter fits

$$
\beta_{c}\left(N_{s}, N_{\tau}\right)=\beta_{c}\left(N_{\tau}\right)+a_{1}\left(N_{\tau}\right) N_{s}^{a_{2}\left(N_{\tau}\right)} .
$$

Inverting the results of these fits defines the deconfining length scale $N_{\tau}\left(\beta_{c}\right)$. Configurations were generated using $2^{19}-2^{25}$ MCOR sweeps, which were partitioned into 32 or more bins. Error bars are then calculated using the jackknife procedure on these bins.

The reweighting curve for the susceptibility is rather flat near the maximum and has large error bars, as shown in figure 1 (left). Nevertheless the error bar on the position of the pseudocritical beta is relatively small. This is explained by the fact that the error bars of figure 1 (left) are strongly correlated, since they are reweighted from the same simulation. Dividing out the maximum susceptibility in each jackknife bin gives us figure 1 (right), which makes the rather small error bar of $\beta_{\max }$ plausible.
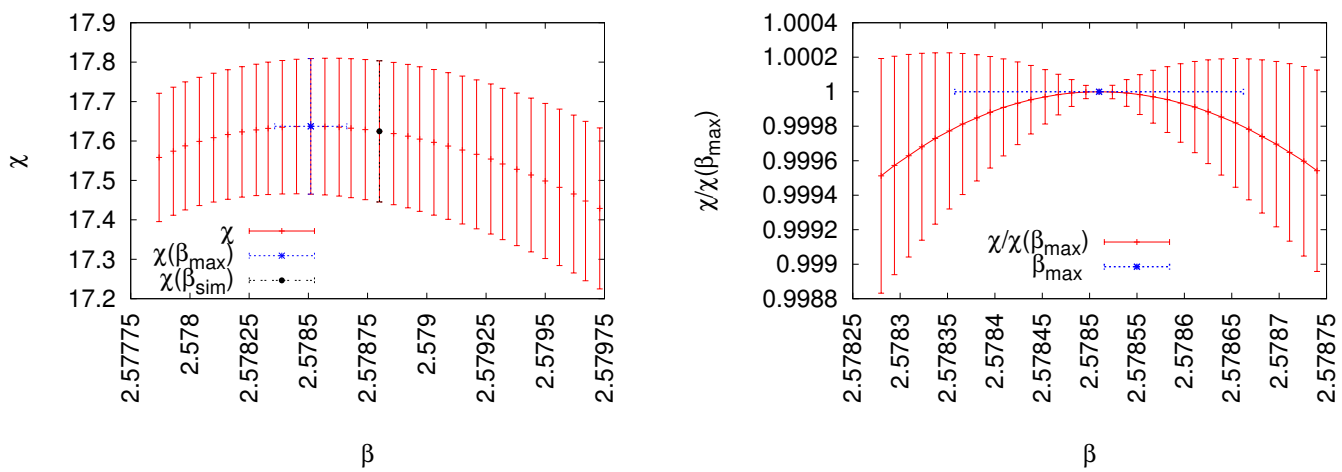

Figure 1. Left: Reweighted susceptibility curve on a $64^{3} \times 10$ lattice simulated at $\beta=2.5788$. Right: Susceptibility curve with maximum value divided out. 


\subsection{Gradient scale}

The gradient flow equation [1]

$$
\dot{V}_{\mu}(x, t)=-g^{2} V_{\mu}(x, t) \partial_{x, \mu} S[V(t)]
$$

is an evolution equation in the fictitious flow time $t$ that decreases the action as $t$ increases. The SU(2) link derivatives are defined by

$$
\left.\partial_{x, \mu} f(V) \equiv i \sum_{i=1}^{3} \sigma_{i} \frac{d}{d s} f\left(e^{i s X^{i}} V\right)\right|_{s=0}, \quad X^{i}\left(x^{\prime}, \mu^{\prime}\right)= \begin{cases}\sigma^{i} & \text { if }\left(x^{\prime}, \mu^{\prime}\right)=(x, \mu), \\ 0 & \text { otherwise }\end{cases}
$$

and the initial condition is $U_{\mu}(x, 0)=U_{\mu}(x)$. The functions

$$
y_{i}(t)=t^{2} E_{i}(t), \quad i=0,1,4
$$

are used to define gradient scales by choosing appropriate fixed target values $y_{i}$ and integrating the gradient flow equation until eq. (8) is satisfied. As a function of $\beta$, the observable

$$
s_{i}(\beta)=\sqrt{t_{i}(\beta)}
$$

scales like a length provided that

1. lattice sizes are chosen so that $N_{\min } \gg \sqrt{8} s_{i}$, where $\sqrt{8} s_{i}$ is the smoothing range [1] and $N_{\text {min }}=\min \left\{N_{i}: i=1,2,3,4\right\}$ for simulations on a $N_{1} N_{2} N_{3} N_{4}$ lattice;

2. the values of $\beta$ are large enough to be in the SU(2) scaling region; and

3. the target values are large enough so that $\sqrt{8} s_{i} \gg 1$ for the smallest used flow time.

One can choose target values so that initial estimates of the scales $s_{i}$ agree with the deconfinement scale for low values of $\beta$. For example figure 2 (left) plots the ratio of $s_{i}(N=12, \beta=2.43$ ) to $s_{i}(N=8, \beta=2.30)$ against $y_{i}$. Due to scaling, one expects this ratio to agree with the ratio of the deconfinement scales, $N_{\tau}(2.43) / N_{\tau}(2.3)=12 / 8=1.5$. This leads to two intersections, one coming from the $E_{4}$ curve and another coming from the $E_{0}$ and $E_{1}$ curves, which practically agree. Figure 2
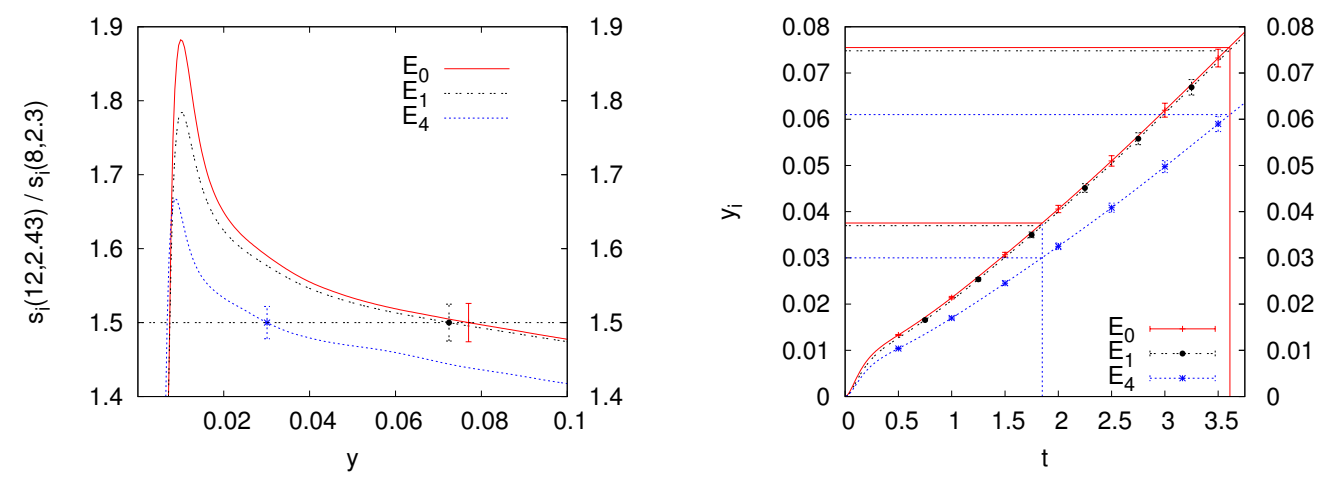

Figure 2. Left: Gradient flow ratios as function of $y$. Right: Gradient flow of an $8^{4}$ lattice at $\beta=2.3$. 
(right) plots the function $t^{2} E_{i}$ against the flow time. Picking initially $y_{4}^{1}=0.030$, the target value corresponding to the aforementioned $E_{4}$ intersection, defines a flow time, indicated by the vertical dotted blue line at $t=1.85$. This flow time is then used to define two more target values $y_{0}^{1}$ and $y_{1}^{1}$ using that figure. Similarly, picking initially $y_{0}^{2}=0.0755$ (or equivalently $y_{1}^{2}=0.0748$ ) delivers three more targets. Hence, we consider altogether six target values:

$$
\begin{aligned}
& y_{0}^{1}=0.0376, \quad y_{1}^{1}=0.0370, \quad y_{4}^{1}=0.030 \\
& y_{0}^{2}=0.0755, \quad y_{1}^{2}=0.0748, \quad y_{4}^{2}=0.061
\end{aligned}
$$

\subsection{Cooling scale}

The cooling algorithm was introduced as part of an investigation of topological charge in the 2D O(3) sigma model [2]. Since then it has found many applications [10]. Bonati and D'Elia showed that using cooling as a smoothing technique produces similar results for topological observables as the gradient flow [3]. An SU(2) cooling step updates a link variable by

$$
V_{\mu}\left(x, n_{c}\right)=\frac{V_{\mu}^{\sqcup}\left(x, n_{c}-1\right)}{\left|V_{\mu}^{\sqcup}\left(x, n_{c}-1\right)\right|},
$$

where $n_{c}$ is the number of cooling steps and $V_{\mu}^{\sqcup}(x)$ is the staple matrix

$$
V_{\mu}^{\sqcup}(x)=\sum_{v \neq \mu}\left[V_{\nu}(x) V_{\mu}(x+\hat{v}) V_{\nu}^{\dagger}(x+\hat{\mu})+V_{v}^{\dagger}(x-\hat{v}) V_{\mu}(x-\hat{v}) V_{\mu}(x-\hat{v}+\hat{\mu})\right] .
$$

The update (12) minimizes the local contribution to the action, so that the "cooling flow" decreases the action. A cooling scale is then defined in the same way as a gradient scale through eq. (9) by iterating eq. (12) until a specified target value is reached. In $4 \mathrm{D}, n_{c}$ cooling steps correspond to a gradient flow time $t_{c}=n_{c} / 3$ [3]; therefore the cooling flow attains its target value at least 34 times faster than the gradient flow, assuming eq. (6) is integrated using the Runge-Kutta scheme with $\epsilon=0.01$. Matching cooling scale ratios to deconfinement scale ratios, the target values are

$$
\begin{aligned}
& y_{0}^{1}=0.0440, \quad y_{1}^{1}=0.0430, \quad y_{4}^{1}=0.0350 \\
& y_{0}^{2}=0.0822, \quad y_{1}^{2}=0.0812, \quad y_{4}^{2}=0.0656
\end{aligned}
$$

\section{Data generation for gradient and cooling scales}

Lattice sizes and $\beta$ values used are given in table 1. In each run, 128 configurations were generated, and on each configuration the gradient and cooling flows were performed. We allocated approximately equal amounts of CPU time to configuration generation and to smoothing flow. Subsequent configurations are separated by $2^{11}-2^{13}$ MCOR sweeps, depending on how many sweeps are needed to reach the target value. Error bars are calculated using the jackknife method with respect to the 128 configurations.

Integrated autocorrelation times $\tau_{\text {int }}$ for the series of configurations are estimated using the software of reference [11] and are found to be statistically compatible with 1. Additionally we calculated for each configuration the cooling flow of the topological charge defined as in reference [3]. An example of cooling trajectories for the 128 configurations is given in figure 3 . Topological correlations are then estimated by calculating $\tau_{\text {int }}$ for the series of topological charges, which are also found to be statistically compatible with 1 . Thus we treat our scale measurements as statistically independent. 
Table 1. Lattice size used to generate data at each $\beta$ value.

\begin{tabular}{ll}
\hline Lattice Size & $\beta$ values \\
\hline $16^{4}$ & 2.300 \\
$28^{4}$ & $2.430,2.510$ \\
$40^{4}$ & $2.574,2.620,2.670,2.710,2.751$ \\
$44^{4}$ & 2.816 \\
$52^{4}$ & 2.875 \\
\hline
\end{tabular}

\section{Scaling and asymptotic scaling analysis}

We analyze 13 length scales: the deconfinement scale $L_{0}$, the gradient scales $L_{1}-L_{6}$ and the cooling scales $L_{7}-L_{12}$. Our goals are to determine whether using cooling scales over gradient scales leads to a significant loss of accuracy; estimate scaling violations; investigate whether the choice of target value leads to seriously distinct scaling behavior; compare scaling and asymptotic scaling fit forms; and finally give an overall estimate of systematic error picked up from reference scale choice and choice of fitting form for continuum limit extrapolations.

We first examine $O\left(a^{2}\right)$ scaling corrections to ratios of lengths. These are fitted using

$$
R_{i, j} \equiv \frac{L_{i}}{L_{j}} \approx r_{i, j}+k_{i, j} a^{2} \Lambda_{L}^{2}=r_{i, j}+c_{i, j}\left(\frac{1}{L_{j}}\right)^{2},
$$

where $r_{i, j}$ is the continuum limit value and $k_{i, j}$ and $c_{i, j}$ are constants. In the following we fix $L_{j}=L_{10}$ and plot the normalized ratio

$$
\frac{R_{i, 10}}{r_{i, 10}}=1+c_{i, 10}^{\prime}\left(\frac{1}{L_{10}}\right)^{2}
$$

in figure 4 (left). We find no discernible loss of accuracy using the cooling scale over the gradient scale, which one can see from relative sizes of error bars in this plot. There is clear overlap between

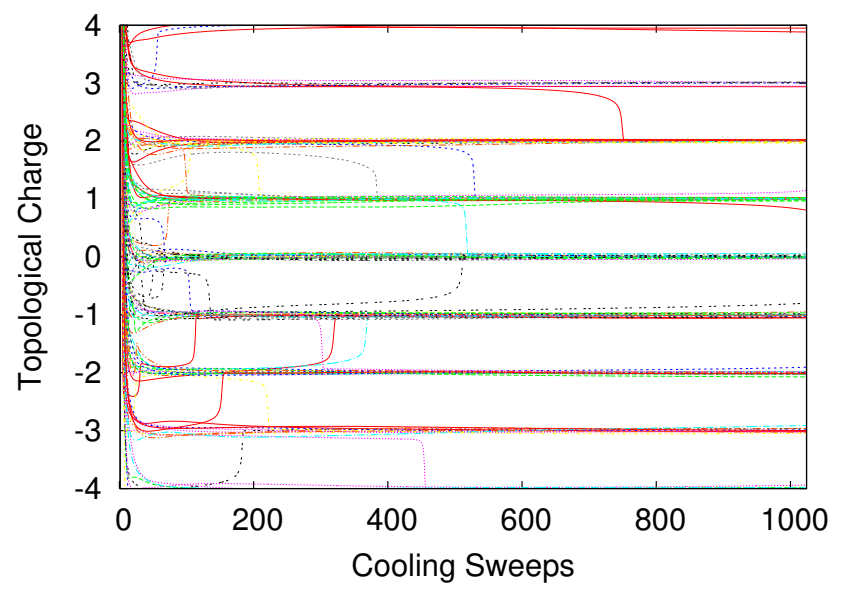

Figure 3. Cooling of the topological charge on a $44^{4}$ lattice at $\beta=2.816$. 

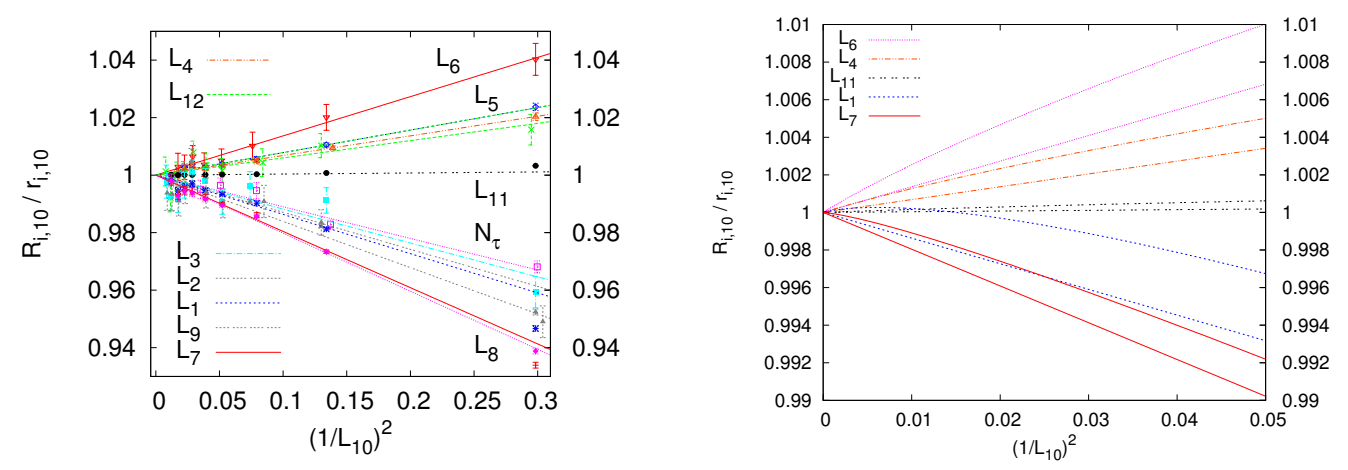

Figure 4. Left: Scaling corrections for ratios $L_{i} / L_{10}$. Some labels are attached to lines and others are in the legend. Here and in the remaining plots the top-bottom ordering of the legend matches the top-bottom ordering of the plot, and some data are shifted slightly for visibility. Right: Direct comparison between representative scaling fits from the left plot and asymptotic scaling fits in the same region. Slightly curved fits of the pairs belong to the asymptotic scaling form, and the data are omitted for visibility.

Table 2. Estimates of the continuum limits of the $L_{i} / L_{j}$ ratios.

\begin{tabular}{lllll}
\hline$i \backslash j$ & $L_{1}$ & $L_{4}$ & $L_{7}$ & $L_{10}$ \\
\hline$L_{0}$ & $2.8896(71)$ & $2.2290(46)$ & $2.8855(68)$ & $2.2618(42)$ \\
$L_{1}$ & & $0.77382(61)$ & $0.99845(38)$ & $0.78433(43)$ \\
$L_{3}$ & $0.9250(19)$ & $0.7163(17)$ & $0.9241(19)$ & $0.7264(16)$ \\
$L_{4}$ & $1.2943(11)$ & & $1.29135(99)$ & $1.01520(49)$ \\
$L_{6}$ & $1.2090(26)$ & $0.9346(20)$ & $1.2081(27)$ & $0.9490(21)$ \\
$L_{7}$ & $1.00156(38)$ & $0.77398(79)$ & & $0.78570(50)$ \\
$L_{9}$ & $0.9222(21)$ & $0.7141(19)$ & $0.9213(20)$ & $0.7243(17)$ \\
$L_{10}$ & $1.27509(70)$ & $0.98508(47)$ & $1.27300(80)$ & \\
$L_{12}$ & $1.1835(24)$ & $0.9164(21)$ & $1.1825(24)$ & $0.9292(19)$ \\
\hline
\end{tabular}

cooling and gradient scales, for example cooling scales $L_{10}-L_{12}$ fall within the spread of gradient scales $L_{1}-L_{6}$, which shows that cooling scales do not suffer significant scaling violations compared to gradient scales. At $\left(1 / L_{10}\right)^{2} \approx 0.30$, which corresponds to $\beta=2.300$, we read off scaling violations of about $10 \%$. Deeper in the scaling region at $\left(1 / L_{10}\right)^{2} \approx 0.05$, which corresponds to $\beta=2.574$, the violations are less than $2 \%$. Results for all our $L_{i} / L_{j}$ continuum estimates are compiled in table 2.

The three-loop asymptotic scaling relation for $\mathrm{SU}(2)$ is

$$
a \Lambda_{L} \approx \exp \left(-\frac{1}{2 b_{0} g^{2}}\right)\left(b_{0} g^{2}\right)^{-b_{1} / 2 b_{0}^{2}}\left(1+q_{1} g^{2}\right) \equiv f_{a s}^{1}(\beta),
$$

where $b_{0}=11 / 24 \pi^{2}[12,13]$ and $b_{1}=17 / 96 \pi^{4}[14,15]$ are the regularization scheme independent coefficients and $q_{1}=0.08324$ [16] using lattice regularization. The superscript of $f_{a s}^{1}$ indicates that only the $O\left(g^{2}\right)$ correction to the asymptotic scaling relation is included. Allton suggested [4] using eq. (18) to fit the approach to the continuum limit by expanding in powers of $a$. For the inverse length 
$1 / L_{i}$ this reads

$$
\frac{1}{L_{i}}=c_{i}^{\prime} a \Lambda_{L}\left(1+\sum_{j=1}^{\infty} \alpha_{i, j}^{\prime}\left(a \Lambda_{L}\right)^{j}\right) .
$$

Plugging eq. (18) into eq. (19) and truncating the series after 3 terms, which is essentially the minimum number needed to get acceptable $q$ values for asymptotic scaling fits, one finds

$$
L_{i} \approx \frac{c_{i}}{f_{a s}^{1}(\beta)}\left(1+\sum_{j=1}^{3} \alpha_{i, j}\left[f_{a s}^{1}(\beta)\right]^{j}\right) .
$$

Due to the $\alpha_{i, 1}$ term in eq. (20) corrections to ratios would in general be of order $a$ in the lattice spacing. This may be the main reason why Allton's approach never became popular. In [17] this problem was avoided by combining the scales discussed there into a single fit, which is only possible if the relative scaling violations are so weak that they can be neglected within the statistical errors. In [5] we relaxed this to the requirement that the $\alpha_{i, 1}$ coefficients have to agree for all scales, i.e. $\alpha_{i, 1} \equiv \alpha_{1}$, which provides a general solution to the problem. The master coefficient was then determined by the maximum likelihood approach, varying $\alpha_{1}$ and repeating all fits for each value. Figure 5 plots eq. (20) against $\beta$, with the asymptotic scaling behavior divided out. With this normalization the curves approach 1 in the continuum limit. At $\beta=2.300$ asymptotic scaling violations range from $28 \%$ to $37 \%$. Differences of ratios at this $\beta$ value reach $14 \%$ (from $0.72 / 0.63 \approx 1.14$ ), in agreement with $12 \%$ (from $1.04 / 0.93 \approx 1.12$ ) of the scaling fits at the same $\beta$ value, shown in figure 4 (left).

For a more direct comparison with scaling, we compute ratios of gradient and cooling lengths using asymptotic scaling. Since all scales have the same first order coefficient, they cancel in the ratio, leading to normalized ratio functions

$$
\frac{R_{i, 10}}{r_{i, 10}}=1+\frac{1}{r_{i, 10}} \sum_{j=2}^{3} \kappa_{i, j}\left[f_{a s}^{1}\right]^{j} .
$$

In figure 4 (right), normalized ratio functions using scaling and asymptotic scaling fits are both plotted against the squared lattice spacing. Straight line fits are scaling fits, while slightly curved fits are asymptotic scaling fits. Systematic error due to choice of fitting form alone seems not to exceed about $0.6 \%$. At $\left(1 / L_{10}\right)^{2}=0.05$, the combined systematic error due to choice of scale, target value, and continuum limit fitting form is read off to be around $2 \%$.

\section{Summary and Conclusions}

A physical scale, here the deconfinement scale, is well suited for determining initial target values for the cooling and gradient flow. The cooling scale is found to reach its target values much faster than the gradient scale without losing accuracy in scale setting. Systematic errors due to using distinct operators, within the same scale, gradient or cooling, are considerably larger than the systematic error encountered by switching from the gradient to the cooling scale or vice versa. For the gradient and cooling scales six target values were used, and the choice of target value gives the largest contribution to the total systematic error, which is approximately $2 \%$ at about $\beta=2.6$. This is small, but larger than the statistical errors of table 2 . Fits relying on asymptotic scaling were compared with the conventionally used scaling fit, and the systematic error between the two fit forms contributes only about $0.6 \%$ to the total systematic error. Our results show that one must probe rather deep in the scaling region to achieve systematic errors of the discussed variety below $1 \%$. 


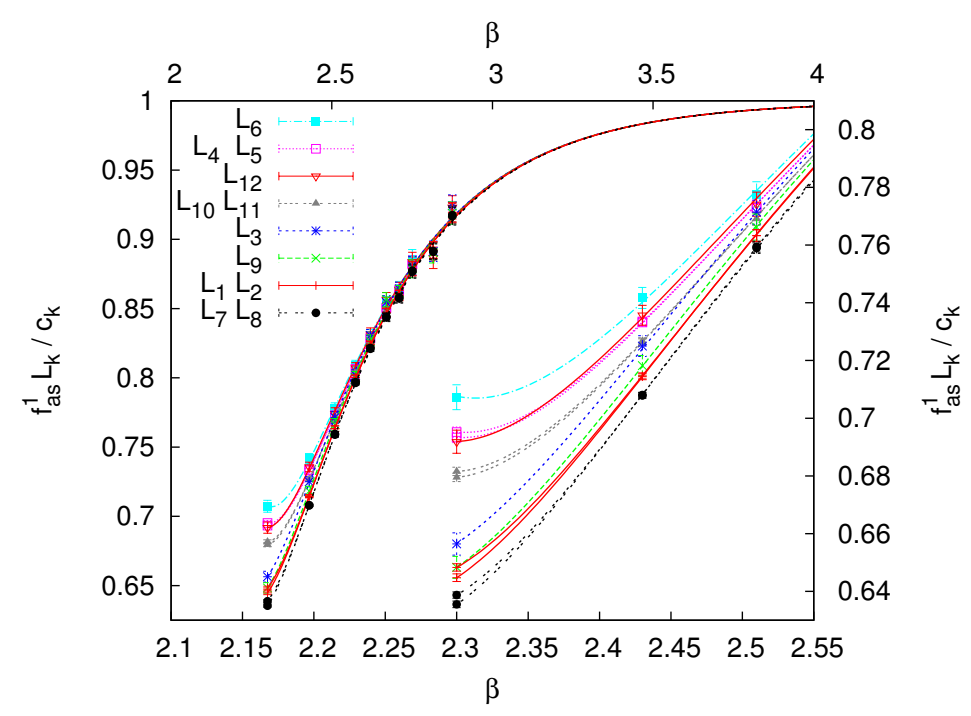

Figure 5. Asymptotic scaling corrections for lengths $L_{k}$. The top abscissa and left ordinate correspond to the top set of curves, while the bottom abscissa and right ordinate correspond to the bottom set of curves, which is an enlargement of the top curves for low $\beta$ values.

Acknowledgements: DC was supported in part by the US Department of Energy (DOE) under Contract No. DE-SC0010102. Our calculations used resources of the National Energy Research Scientific Computing Center, supported by the DOE under Contract No. DE-AC02-05CH11231.

\section{References}

[1] M. Lüscher, JHEP 2010, 1 (2010)

[2] B.A. Berg, Phys. Lett. 104B, 475 (1981)

[3] C. Bonati, M. D’Elia, Phys. Rev. D 89, 105005 (2014)

[4] C.R. Allton, Nucl. Phys. B (Proc. Suppl.) 53, 867 (1997)

[5] B.A. Berg, D.A. Clarke, Phys. Rev. D 95, 094508 (2017)

[6] K. Fabricius, O. Haan, Phys. Lett. B 143, 459 (1984)

[7] A.D. Kennedy, B.J. Pendleton, Phys. Lett. 156B, 393 (1985)

[8] S.L. Adler, Phys. Rev. D 37, 458 (1988)

[9] D. Barkai, K.J.M. Moriarty, Comput. Phys. Commun. 27, 105 (1982)

[10] E. Vicari, H. Panagopoulos, Phys. Rep. 470, 93 (2009)

[11] B. Berg, Markov Chain Monte Carlo Simulations and Their Statistical Analysis (World Scientific, Singapore, 2004)

[12] D.J. Gross, F. Wilczek, Phys. Rev. D 8, 3633 (1973)

[13] H.D. Politzer, Phys. Rev. Lett. 30, 1346 (1973)

[14] W.E. Caswell, Phys. Rev. Lett. 33, 244 (1974)

[15] D.R.T. Jones, Nucl. Phys. B 75, 531 (1974)

[16] B. Allés, A. Feo, H. Panagopoulos, Nucl. Phys. B 491, 498 (1997)

[17] B.A. Berg, Phys. Rev. D 92, 054501 (2015) 